\title{
Symbolic Dynamical Model of Average Queue Size of Random Early Detection Algorithm
}

\author{
Charlotte Yuk-Fan Ho \\ Telephone: +44 (0)20 78825555 ext. 4333 Fax:+44 (0)20 78827997 Email: c.ho@qmul.ac.uk \\ School of Mathematical Sciences, Queen Mary, University of London, Mile End Road, London, E1 4NS, United \\ Kingdom. \\ *Bingo Wing-Kuen Ling \\ Telephone: +44 (0)20 78482294 Fax: +44 (0)20 78482932 Email: wing-kuen.ling@kcl.ac.uk \\ Department of Electronic Engineering, Division of Engineering, King's College London, Strand, London, WC2R 2LS, \\ United Kingdom. \\ Herbert H. C. Iu \\ Telephone: +618 64887989 Fax: +618 64887989 Email: herbert@ee.uwa.edu.au \\ School of Electrical, Electronic and Computer Engineering, The University of Western Australia, 35 Stirling Highway, \\ Crawley, Perth, Western Australia, WA6009, Australia.
}

\begin{abstract}
In this paper, a symbolic dynamical model of the average queue size of the random early detection (RED) algorithm is proposed. The conditions on both the system parameters and the initial conditions that the average queue size of the RED algorithm would converge to a fixed point are derived. These results are useful for network engineers to design both the system parameters and the initial conditions so that internet networks would achieve a good performance.
\end{abstract}

Keywords - Transmission control protocol, random early detection algorithm, internet congestion problem, symbolic dynamics, Lyapunov stability.

\section{INTRODUCTION}

There is no doubt that internet networks play an important role in our daily life. However, as 
International Journal of Bifurcation and Chaos

the traffic of internet networks grows rapidly, congestion problems become very serious. Poor managements of internet networks will result to partly or fully inaccessible networks and hence degrade general performances of networking applications [1]-[6]. To address this issue, various approaches have been proposed. The commonest approach to address the congestion problems is via active queue management (AQM) mechanisms, in which the RED algorithm is a widely deployed algorithm for AQM mechanisms [1]-[6].

The goal of the RED algorithm is to detect an early sign of the congestion and provide a feedback by either dropping or marking segments of messages so that the congestion can be avoided. Although the RED algorithm is conceptually simple, the interaction between the transmission control protocol (TCP) and the RED algorithm at the router's gateway is actually governed by a first order piecewise nonlinear difference equation, in which, complex behaviors, such as limit cycle behaviors and chaotic behaviors, could be exhibited. For the commonest operation, the average queue size of the RED algorithm is required to converge to a fixed point and these complex behaviors degrade general performances of network applications [1]-[6]. As these complex behaviors depend on both the system parameters and the initial conditions of the nonlinear difference equation, network engineers require to design both the system parameters and the initial conditions of the nonlinear difference equation so that the average queue size of the RED algorithm would converge to a fixed point. Nevertheless, no result has been reported on characterizing the conditions on both the system parameters and the initial conditions of the nonlinear difference equation that the average queue size of the RED algorithm would converge to a fixed point. This paper is to address this issue.

The outline of this paper is as follows. The working principles of the TCP and the RED algorithm are reviewed in Section II, while nonlinear behaviors of the average queue size of the RED algorithm are reviewed in Section III. In Section IV, a symbolic dynamical model is proposed as well as the conditions on both the system parameters and the initial conditions of the nonlinear difference equation that the average queue size of the RED algorithm would converge to a fixed 
International Journal of Bifurcation and Chaos

point are derived. Finally, a conclusion is drawn in Section V.

\section{REVIEW ON WORKING PRINCIPLES OF TCP AND RED ALGORITHM}

This section describes a brief summary of the working principles of the TCP and the RED algorithm. For interested readers, please refer to the details in [1]-[6].

\section{A. Working principles of TCP}

The transmission rate of a TCP connection is controlled by the size of the congestion window at the sender end, denoted as cwnd. The cwnd size determines the number of segments of messages to be sent to the receiver end. The $c$ wnd size is adjusted to maximize the utilization of the link and to avoid the congestion. To adjust the cwnd size, TCP congestion control algorithms employ the following four phases: the slow start phase, the congestion avoidance phase, the fast retransmit phase and the fast recovery phase.

The description of the slow start phase is as follows. When a new connection is first established, the cwnd size at the sender end is initialized to the size of one segment of messages. Upon a receipt of every segment of messages, a packet of an acknowledgement (ACK) is sent to the TCP sender by the TCP receiver. Upon a receipt of every packet of an ACK at the sender end, the TCP sender increases the cwnd size by the size of one segment of messages. Two segments of messages can now be sent. When both segments of messages are acknowledged, the cwnd size is increased to the size of four segments of messages. These procedures are iterated in an exponential manner and the TCP sender opens up the window size exponentially, that is, $1 \rightarrow 2 \rightarrow 4 \rightarrow 8, \ldots$, etc.

When the cwnd size exceeds a threshold, denoted as ssthresh, the TCP sender enters the congestion avoidance phase. During the congestion avoidance phase, the cwnd size is incremented by the size of one segment of messages per a round trip time regardless of the number of the packets of an ACK has been received. Hence, the TCP sender opens up the window size linearly, that is, $1 \rightarrow 2 \rightarrow 3 \rightarrow 4, \ldots$, etc, until it reaches the receiver's advertised window size, denoted as rwnd. 
The description of the fast retransmit phase is as follows. A retransmission timer is set every time when the TCP sender sends a packet of messages. A packet loss is detected by the timeout mechanism if the timer expires before receiving the packet of an ACK. In this case, the TCP sender adjusts the ssthresh and switches back to the slow start phase. In the congestion avoidance phase, upon receiving an out of order segment of messages, the TCP receiver generates a packet of an ACK and is immediately followed by a duplicate packet of an ACK. When three duplicate packets of an ACK have been received by the TCP sender, it is assumed that a segment of messages has been lost. The TCP sender halves the cwnd size and retransmits the lost segment of messages without waiting the expiration of a retransmission timer.

The description of the fast recovery phase is as follows. Until the retransmitted segment of messages is received, the TCP receiver will continue to receive the out of order segments of messages and generate the duplicate packets of an ACK to the TCP sender. After the fast retransmit phase sends the missing segment of messages, the TCP sender increases the $c$ wnd size whenever each duplicate packet of an ACK is received. Each duplicate packet of an ACK is an indication that one packet of messages has reached the TCP receiver and the number of outstanding packets of messages has decreased by one. Therefore, the TCP sender is allowed to increment the cwnd size. The TCP sender switches back to the congestion avoidance phase when the retransmitted segment of messages is received and a nonduplicate packet of an ACK is sent to the TCP sender.

\section{B. Working principles of RED algorithm}

The RED algorithm is a gateway based algorithm for AQM mechanisms. It estimates the congestion level by monitoring and updating the average queue size. In order to maintain a relatively small average queue size rather than waiting for buffer overflows, it drops a packet of messages with a certain probability to provide an early sign of the congestion when the average queue size exceeds a threshold. Denote the minimum queue threshold, the maximum queue threshold, the maximum packet drop probability, the average queue size and the drop probability at the $k^{\text {th }}$ iteration $\forall k \geq 0$ as $q_{\min }, q_{\max }, \quad p_{\max }, \bar{q}(k)$ and $p(k)$, respectively. The drop 
International Journal of Bifurcation and Chaos

probability depends on the average queue size and it is governed by the following equation:

$$
p(k)=H(\bar{q}(k)) \equiv\left\{\begin{array}{cc}
0 & 0 \leq \bar{q}(k)<q_{\min } \\
\frac{\bar{q}(k)-q_{\min }}{q_{\max }-q_{\min }} p_{\max } & q_{\min } \leq \bar{q}(k)<q_{\max } \\
1 & q_{\max } \leq \bar{q}(k)
\end{array} \quad \forall k \geq 0 .\right.
$$

Denote the exponential average weight of the RED algorithm as $w$. The average queue size at the $k+1^{\text {th }}$ iteration is governed by an exponential law and it depends on both the average queue size and the drop probability at the $k^{\text {th }}$ iteration $\forall k \geq 0$ as follows:

$$
\bar{q}(k+1)=(1-w) \bar{q}(k)+w G(p(k)),
$$

in which

$$
G(p(k)) \equiv\left\{\begin{array}{cc}
\max \left(\frac{N K}{\sqrt{p(k)}}-\frac{C d}{M}, 0\right) & p(k) \neq 0 \quad \\
N r w n d-\frac{C d}{M} & p(k)=0
\end{array} \quad \forall k \geq 0,\right.
$$

where the capacity of the link between two routers, the packet size, the number of TCP connections, a constant between 1 and $\sqrt{\frac{8}{3}}$ as well as the round trip propagation delay are denoted as $C, M$, $N, K$ and $d$, respectively.

It is trivial to see that the dynamical model of the average queue size can be further represented by a first order piecewise nonlinear difference equation as follows:

$$
\bar{q}(k+1)=\left\{\begin{array}{ccc}
(1-w) \bar{q}(k)+w \max \left(\frac{N K}{\sqrt{p(k)}}-\frac{C d}{M}, 0\right) & p(k) \neq 0 & \\
(1-w) \bar{q}(k)+w\left(N r w n d-\frac{C d}{M}\right) & p(k)=0
\end{array} \quad \forall k \geq 0 .\right.
$$

Due to the physical nature of both the parameters and the variables of the RED algorithm, it is assumed that all the parameters $\left(q_{\min }, q_{\max }, p_{\max }, w, C, M, N, K, d\right.$ and $\left.r w n d\right)$ are nonnegative and real-valued. Also, it is assumed that $0 \leq p_{\max } \leq 1, q_{\min }<q_{\max }, \bar{q}(0) \geq 0$ and $0<w<1$

In this model, the dynamics of the average queue size of the RED algorithm at the gateway is considered. The first order piecewise nonlinear dynamical model reflects the TCP congestion 
International Journal of Bifurcation and Chaos

control mechanism and takes into account the slow start phase and timeout events.

\section{NONLINEAR BEHAVIORS OF AVERAGE QUEUE SIZE OF RED ALGORITHM}

It is well known that the average queue size of the RED algorithm could exhibit a bifurcation behavior. Figure 1 shows the bifurcation diagram as $w$ varies when $N=1, K=\sqrt{\frac{3}{2}}$, $C=1.54 \times 10^{6}, \quad d=0.0228, \quad M=4000, \quad r w n d=1000, \quad q_{\min }=5, \quad q_{\max }=15, \quad p_{\max }=0.1$, $\bar{q}(0)=0$ and $p(0)=0$. Figure 2 shows the frequency spectrum of the steady state drop probability, the steady state phase diagram, the steady state drop probability and the steady state average queue size when $w=0.22$, while Figure 3, Figure 4 and Figure 5 show the corresponding numerical computer simulation results when $w=0.23, w=0.25$ and $w=0.275$, respectively. It can be seen from Figure 1 to Figure 5 that as $w$ increases, the steady state drop probability and the steady state average queue size exhibit the limit cycle and the random like chaotic behaviors consecutively. When $w \geq 0.27$, the steady state drop probability at some time instants is equal to one. Hence, it can be seen from Figure 5 that there are two straight lines, one located at $p(k)=\frac{\bar{q}(k)-q_{\min }}{q_{\max }-q_{\min }} p_{\max }$ and another one located at $p(k)=1$, exhibited on the steady state phase diagram. On the other hand, when $w<0.27$, it can be seen from Figure 3 that there is only one single straight line, located at $p(k)=\frac{\bar{q}(k)-q_{\min }}{q_{\max }-q_{\min }} p_{\max }$, exhibited on the steady state phase diagram. The importance of observing the above nonlinear phenomena is that network engineers could design both the system parameters and the initial conditions so that the average queue size of the RED algorithm would not exhibit these nonlinear behaviors. 
International Journal of Bifurcation and Chaos

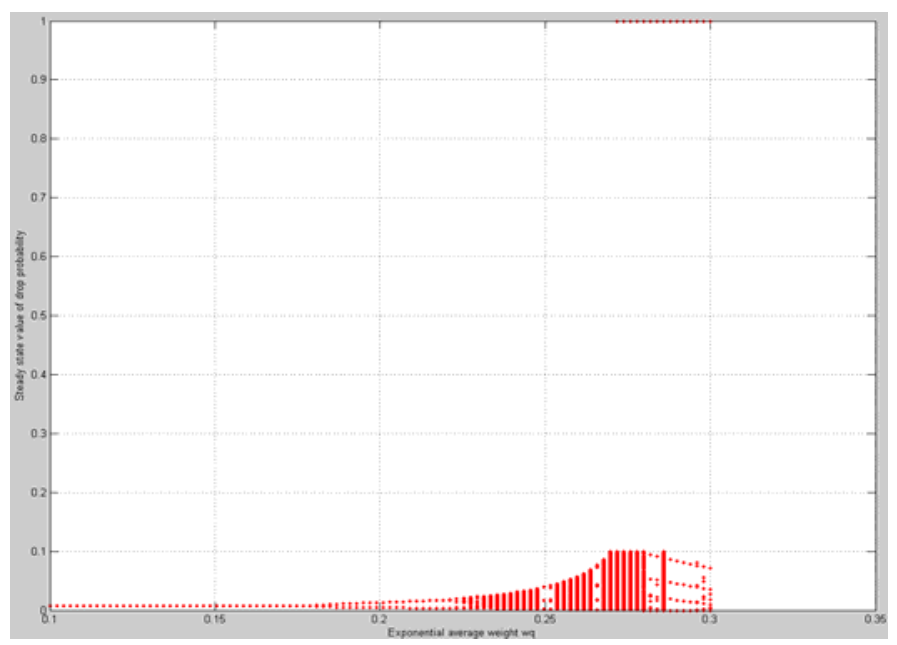

Figure 1. Bifurcation diagram as $w$ varies.
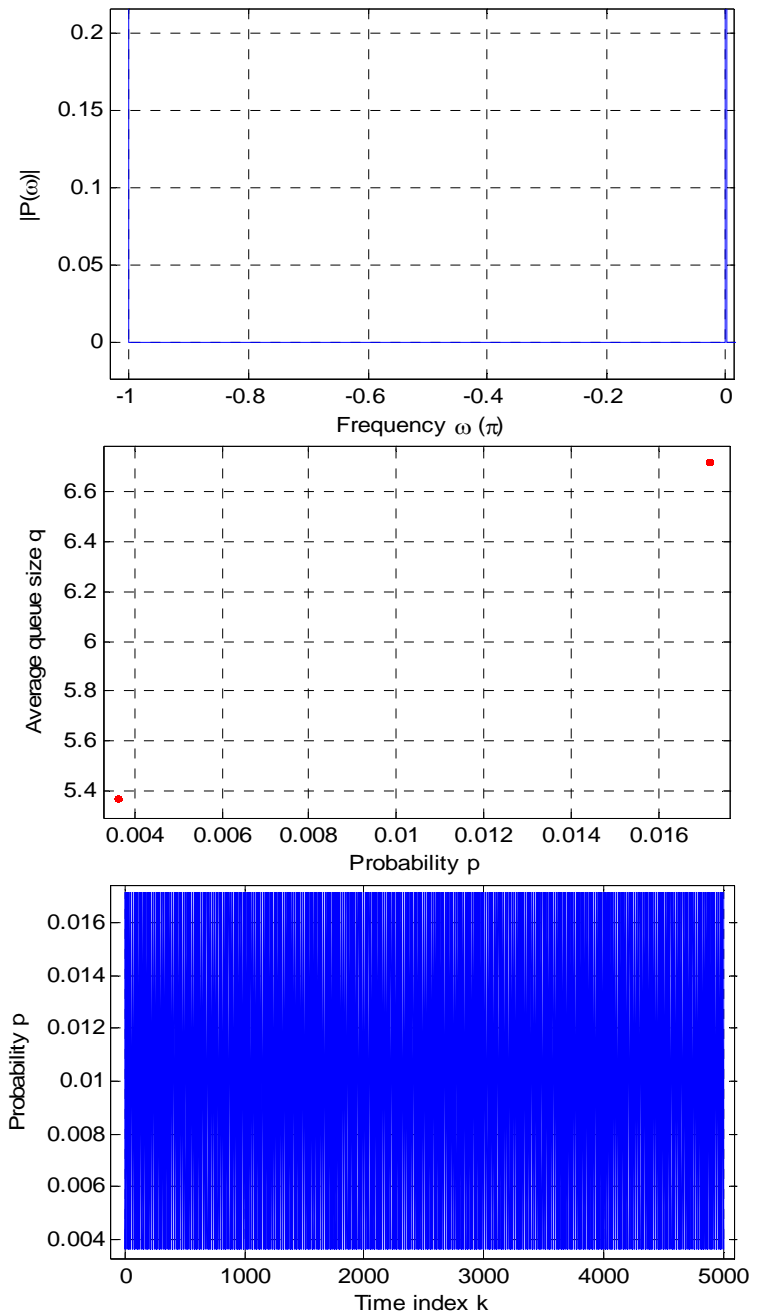
International Journal of Bifurcation and Chaos

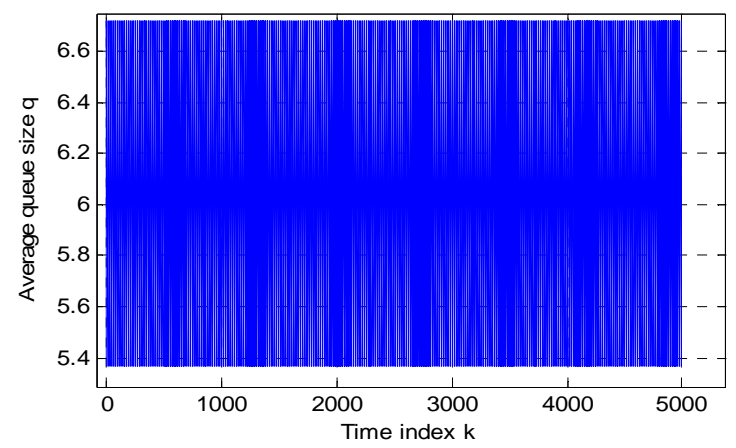

Figure 2. $w=0.22$. (a) Frequency spectrum of the steady state drop probability. (b) Steady state phase diagram. (c) Steady state drop probability. (d) Steady state average queue size.
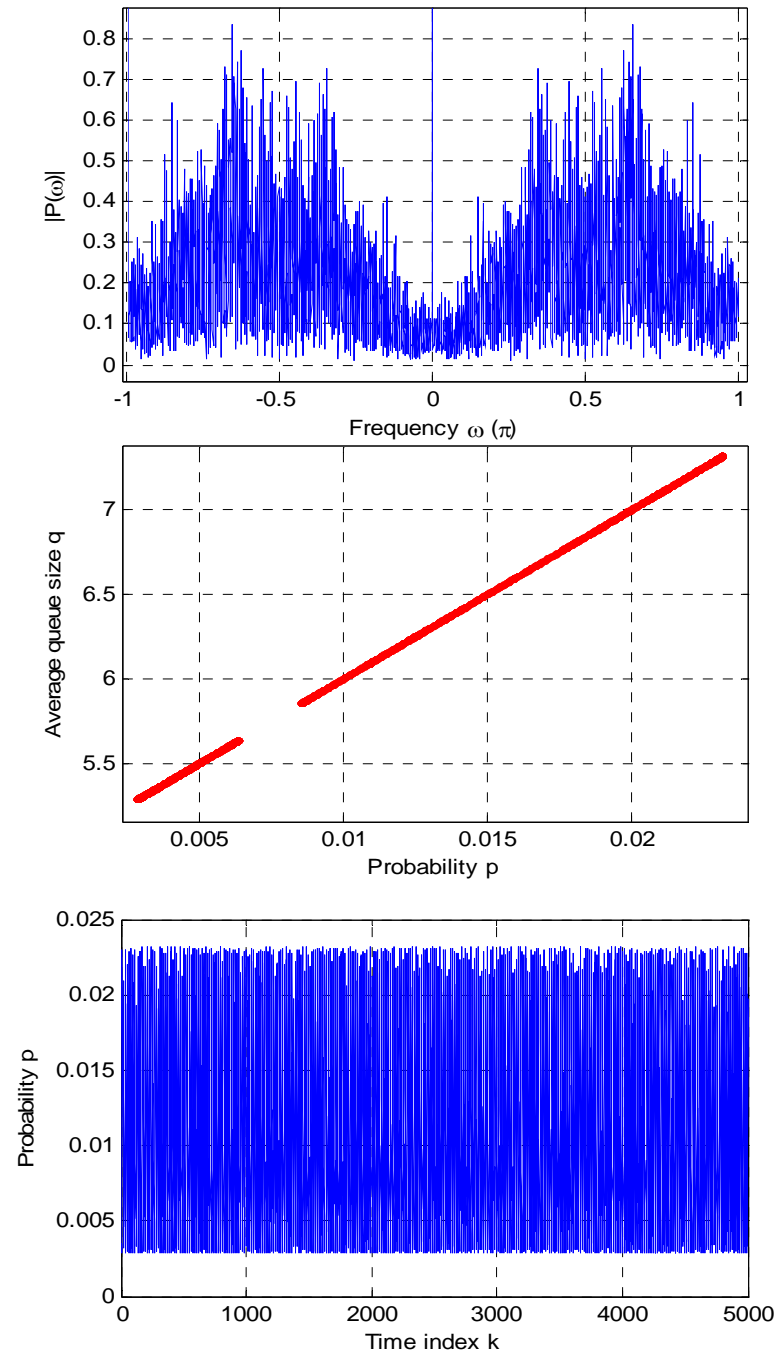
International Journal of Bifurcation and Chaos

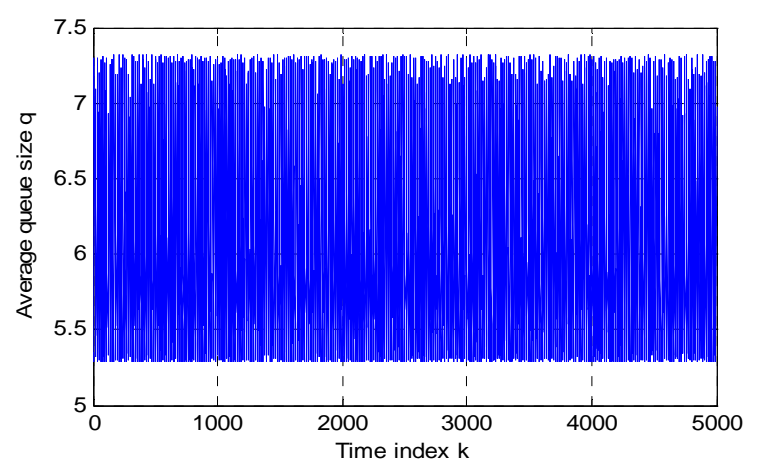

Figure 3. $w=0.23$. (a) Frequency spectrum of the steady state drop probability. (b) Steady state phase diagram. (c) Steady state drop probability. (d) Steady state average queue size.
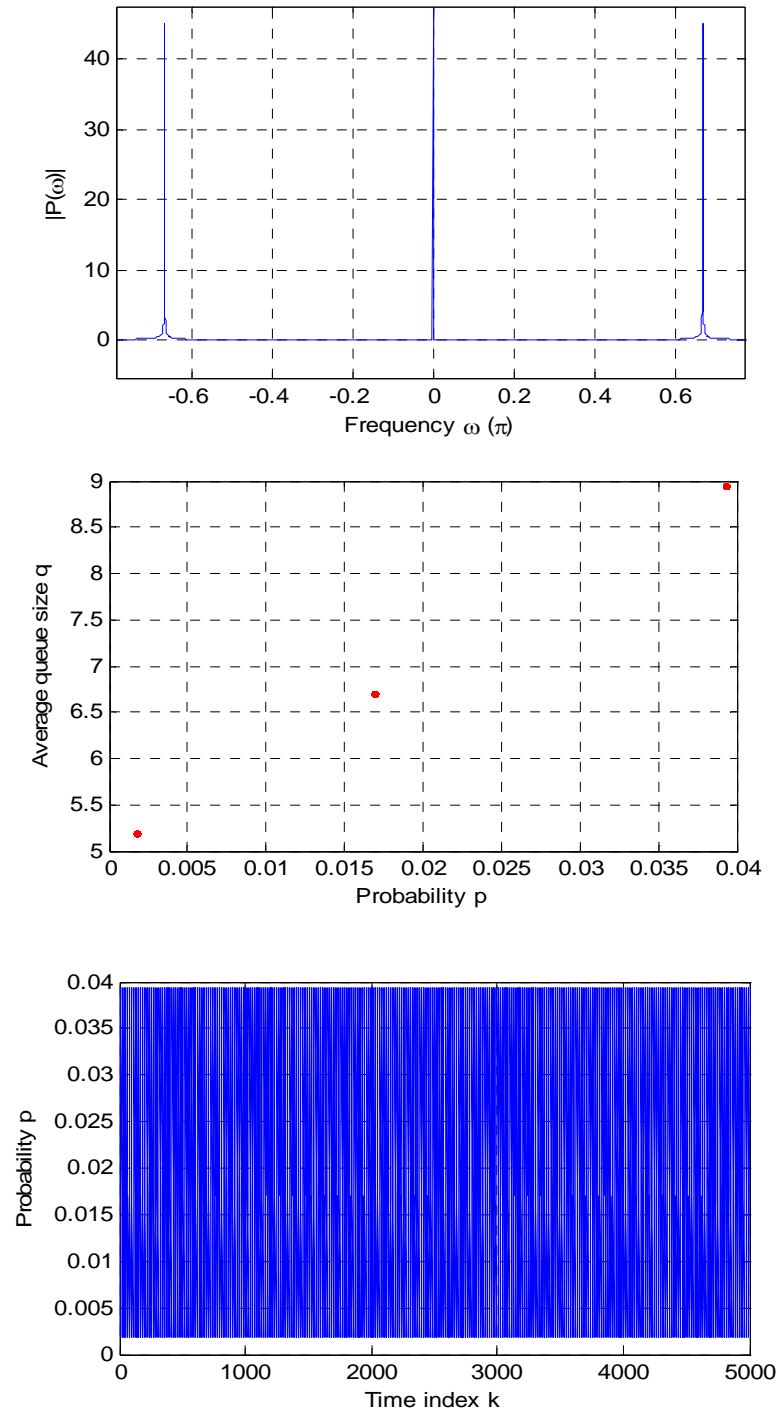
International Journal of Bifurcation and Chaos

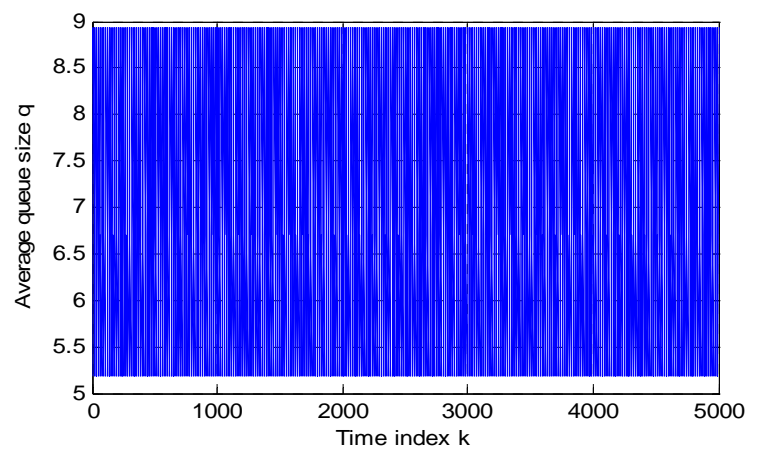

Figure 4. $w=0.25$. (a) Frequency spectrum of the steady state drop probability. (b) Steady state phase diagram. (c) Steady state drop probability. (d) Steady state average queue size.
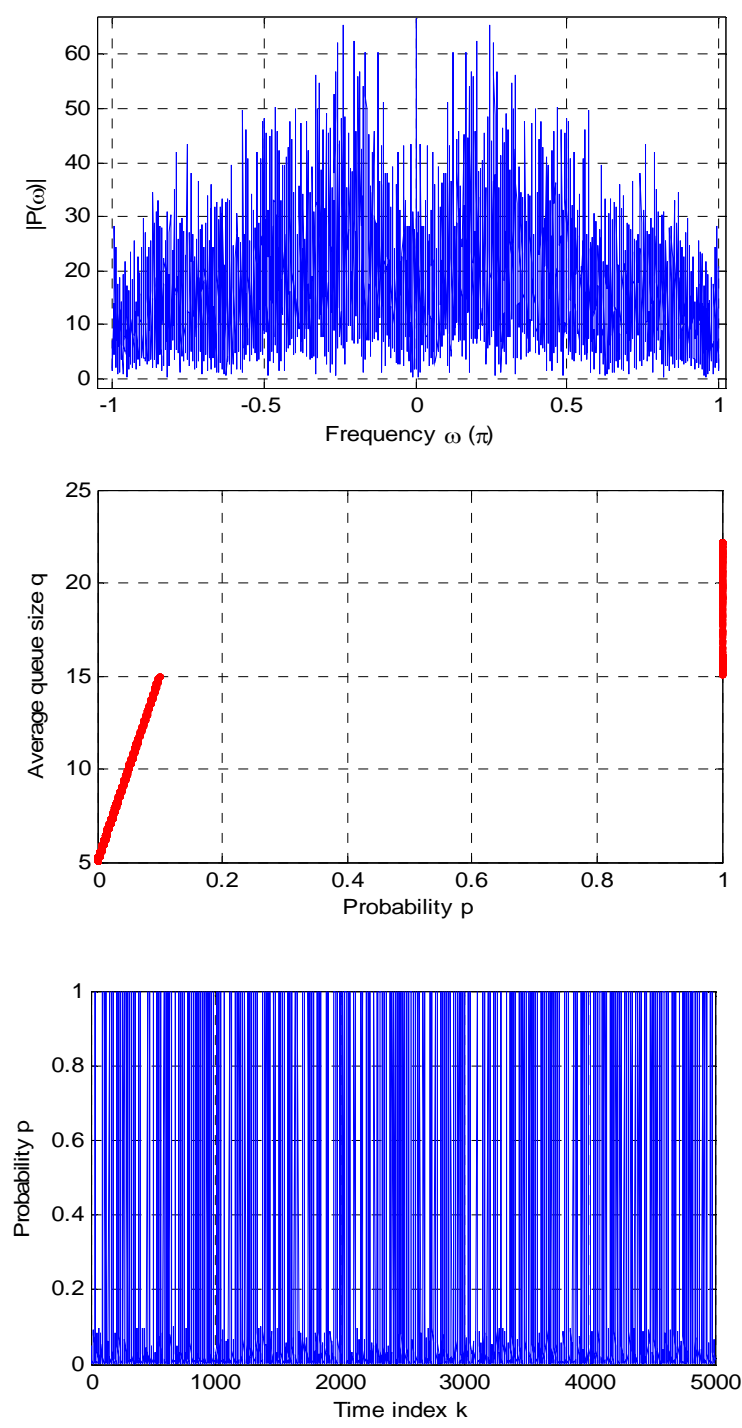
International Journal of Bifurcation and Chaos

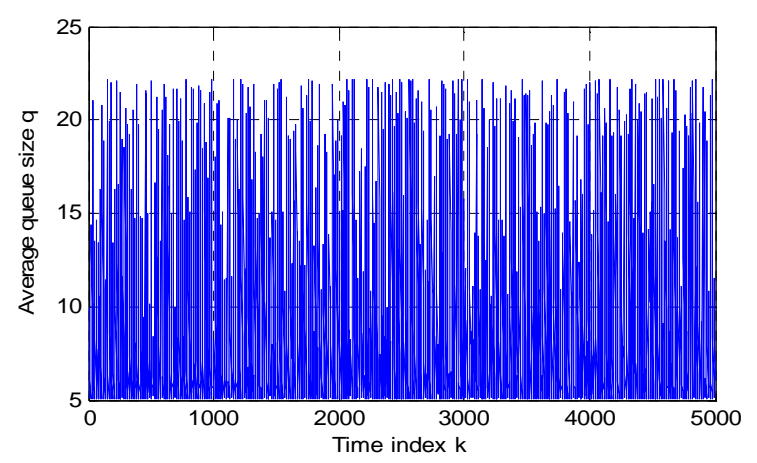

Figure 5. $w=0.275$. (a) Frequency spectrum of the steady state drop probability. (b) Steady state phase diagram. (c) Steady state drop probability. (d) Steady state average queue size.

\section{SYMBOLIC DYNAMICAL MODEL AND CONDITIONS FOR EXHIBITING FIXED POINT BEHAVIORS}

A. Symbolic dynamical model

It is obvious to see that different values of $\bar{q}(k)$ corresponds to different dynamical equations. To analyze the behaviors of the average queue size of the RED algorithm, the set of $\bar{q}(k)$ is partitioned into fifteen different subsets, denoted as $S_{i}$ for $i=1, \cdots, 15$. These subsets are represented by fifteen different symbols, denoted as $s_{i}(k)$ for $i=1, \cdots, 15$ and $\forall k \geq 0$, in which only one symbol is activated in each subset. That is, if $\bar{q}(k) \in S_{i}$, then $s_{i}(k)=1$ and $s_{j}(k)=0$ for $j \neq i$ and $\forall k \geq 0$. Denote $\mathbf{s}(k)=\left[\begin{array}{lll}s_{1}(k) & \cdots & s_{15}(k)\end{array}\right]^{T} \quad \forall k \geq 0$, where the superscript ${ }^{T}$ denotes the transpose operator. The model of the average queue size of the RED algorithm can be analyzed via a symbolic dynamical model, where symbolic dynamics is a system dynamics in which some signals in the system are multileveled. The dynamics of the average queue size of the RED algorithm in each subset is as follows:

A.1 Dynamics of the average queue size in the first subset

$$
\text { If } 0 \leq \bar{q}(k)<q_{\min } \text { and } \frac{w\left(\frac{C d}{M}-N r w n d\right)}{1-w} \leq \bar{q}(k)<\frac{q_{\min }-w\left(N r w n d-\frac{C d}{M}\right)}{1-w} \text {, then } p(k)=0 \text {, }
$$


International Journal of Bifurcation and Chaos

$\bar{q}(k+1)=(1-w) \bar{q}(k)+w\left(N r w n d-\frac{C d}{M}\right)$ and $p(k+1)=0$. Denote $s_{1}(k)=1$ and $s_{j}(k)=0$ for $j \neq 1$.

Proof:

It is obvious to see that if $0 \leq \bar{q}(k)<q_{\min }$, then $p(k)=0$. Hence, we have $\bar{q}(k+1)=(1-w) \bar{q}(k)+w\left(N r w n d-\frac{C d}{M}\right)$. As $\frac{w\left(\frac{C d}{M}-N r w n d\right)}{1-w} \leq \bar{q}(k)<\frac{q_{\min }-w\left(N r w n d-\frac{C d}{M}\right)}{1-w}$, we have $0 \leq(1-w) \bar{q}(k)+w\left(N r w n d-\frac{C d}{M}\right)<q_{\min }$. This implies that $0 \leq \bar{q}(k+1)<q_{\min }$. Hence, we have $p(k+1)=0$. This completes the proof.

A.2 Dynamics of the average queue size in the second subset

If $\quad 0 \leq \bar{q}(k)<q_{\min } \quad$ and $\quad \bar{q}(k) \geq \frac{q_{\max }-w\left(N r w n d-\frac{C d}{M}\right)}{1-w}$, then $p(k)=0$, $\bar{q}(k+1)=(1-w) \bar{q}(k)+w\left(N r w n d-\frac{C d}{M}\right)$ and $p(k+1)=1$. Denote $s_{2}(k)=1$ and $s_{j}(k)=0$ for $j \neq 2$.

Proof:

It is obvious to see that if $0 \leq \bar{q}(k)<q_{\min }$, then $p(k)=0$. Hence, we have $\bar{q}(k+1)=(1-w) \bar{q}(k)+w\left(N r w n d-\frac{C d}{M}\right)$. As $\quad \bar{q}(k) \geq \frac{q_{\max }-w\left(N r w n d-\frac{C d}{M}\right)}{1-w}$, we have $(1-w) \bar{q}(k)+w\left(N r w n d-\frac{C d}{M}\right) \geq q_{\max }$. This implies that $\bar{q}(k+1) \geq q_{\max }$. Hence, we have $p(k+1)=1$. This completes the proof.

A.3 Dynamics of the average queue size in the third subset

If $0 \leq \bar{q}(k)<q_{\min }$ and $\frac{q_{\max }-w\left(N r w n d-\frac{C d}{M}\right)}{1-w}>\bar{q}(k) \geq \frac{q_{\min }-w\left(N r w n d-\frac{C d}{M}\right)}{1-w}$, then $p(k)=0, \quad \bar{q}(k+1)=(1-w) \bar{q}(k)+w\left(N r w n d-\frac{C d}{M}\right)$ and 
International Journal of Bifurcation and Chaos

$$
p(k+1)=\frac{(1-w) \bar{q}(k)+w\left(N r w n d-\frac{C d}{M}\right)-q_{\min }}{q_{\max }-q_{\min }} p_{\max } .
$$

Denote $s_{3}(k)=1$ and $s_{j}(k)=0$ for $j \neq 3$.

Proof:

It is obvious to see that if $0 \leq \bar{q}(k)<q_{\min }$, then $p(k)=0$. Hence, we have $\bar{q}(k+1)=(1-w) \bar{q}(k)+w\left(N r w n d-\frac{C d}{M}\right)$. As $\frac{q_{\max }-w\left(N r w n d-\frac{C d}{M}\right)}{1-w}>\bar{q}(k) \geq \frac{q_{\min }-w\left(N r w n d-\frac{C d}{M}\right)}{1-w}$, we have $q_{\max }>(1-w) \bar{q}(k)+w\left(N r w n d-\frac{C d}{M}\right) \geq q_{\min }$. This implies that $q_{\max }>\bar{q}(k+1) \geq q_{\min }$. Hence, we have $p(k+1)=\frac{\bar{q}(k+1)-q_{\min }}{q_{\max }-q_{\min }} p_{\max }=\frac{(1-w) \bar{q}(k)+w\left(N r w n d-\frac{C d}{M}\right)-q_{\min }}{q_{\max }-q_{\min }} p_{\max }$. This completes the proof.

A.4 Dynamics of the average queue size in the fourth subset

$$
\text { Denote } P_{4} \equiv\left\{\begin{array}{l}
p(k): \frac{(1-w)\left(q_{\max }-q_{\min }\right)}{p_{\max }}(p(k))^{\frac{3}{2}}+w N K+\left((1-w) q_{\min }-\frac{w C d}{M}-q_{\min }\right)(p(k))^{\frac{1}{2}}<0 \\
\text { and } \frac{(1-w)\left(q_{\max }-q_{\min }\right)}{p_{\text {max }}}(p(k))^{\frac{3}{2}}+w N K+\left((1-w) q_{\min }-\frac{w C d}{M}\right)(p(k))^{\frac{1}{2}} \geq 0
\end{array}\right\}
$$

and $\quad Q_{4} \equiv\left\{\bar{q}(k): \bar{q}(k)=\frac{q_{\max }-q_{\min }}{p_{\max }} p(k)+q_{\min }\right.$ and $\left.p(k) \in P_{4}\right\} \quad$. If $\quad q_{\min } \leq \bar{q}(k)<q_{\max }$, $\bar{q}(k) \leq \frac{q_{\max }-q_{\min }}{p_{\max }}\left(\frac{M N K}{C d}\right)^{2}+q_{\min } \quad$ and $\quad \bar{q}(k) \in Q_{4} \quad, \quad$ then $\quad p(k)=\frac{\bar{q}(k)-q_{\min }}{q_{\max }-q_{\min }} p_{\max }$, $\bar{q}(k+1)=(1-w) \bar{q}(k)+w\left(\frac{N K}{\sqrt{\frac{\bar{q}(k)-q_{\min }}{q_{\max }-q_{\min }} p_{\max }}}-\frac{C d}{M}\right) \quad$ and $\quad p(k+1)=0$. Denote $\quad s_{4}(k)=1 \quad$ and $s_{j}(k)=0$ for $j \neq 4$.

Proof: 
International Journal of Bifurcation and Chaos

It is obvious to see that if $q_{\min } \leq \bar{q}(k)<q_{\max }$, then $p(k)=\frac{\bar{q}(k)-q_{\min }}{q_{\max }-q_{\min }} p_{\max }$. Since $\bar{q}(k) \leq \frac{q_{\max }-q_{\min }}{p_{\max }}\left(\frac{M N K}{C d}\right)^{2}+q_{\min }$, this implies that $\frac{\bar{q}(k)-q_{\min }}{q_{\max }-q_{\min }} p_{\max } \leq\left(\frac{M N K}{C d}\right)^{2}, \quad p(k) \leq\left(\frac{M N K}{C d}\right)^{2}$, $\frac{C d}{K} \leq \frac{M N}{\sqrt{p(k)}}$ and $\frac{M N}{\sqrt{p(k)}}-\frac{C d}{K} \geq 0$. Hence, we have

$$
\bar{q}(k+1)=(1-w) \bar{q}(k)+w\left(\frac{N K}{\sqrt{\frac{\bar{q}(k)-q_{\min }}{q_{\max }-q_{\min }} p_{\max }}}-\frac{C d}{M}\right) .
$$

As $\bar{q}(k) \in Q_{4}$, we have $p(k) \in P_{4}$. In other words, we have

$$
\frac{(1-w)\left(q_{\max }-q_{\min }\right)}{p_{\max }}(p(k))^{\frac{3}{2}}+w N K+\left((1-w) q_{\min }-\frac{w C d}{M}-q_{\min }\right)(p(k))^{\frac{1}{2}}<0
$$

and

$$
\frac{(1-w)\left(q_{\max }-q_{\min }\right)}{p_{\max }}(p(k))^{\frac{3}{2}}+w N K+\left((1-w) q_{\min }-\frac{w C d}{M}\right)(p(k))^{\frac{1}{2}} \geq 0 .
$$

This implies that

$$
\frac{(1-w)\left(q_{\max }-q_{\min }\right)}{p_{\max }} p(k)+\frac{w N K}{\sqrt{p(k)}}+(1-w) q_{\min }-\frac{w C d}{M}-q_{\min }<0
$$

and

$$
\frac{(1-w)\left(q_{\max }-q_{\min }\right)}{p_{\max }} p(k)+\frac{w N K}{\sqrt{p(k)}}+(1-w) q_{\min }-\frac{w C d}{M} \geq 0,
$$

as well as

$$
(1-w)\left(\frac{q_{\max }-q_{\min }}{p_{\max }} p(k)+q_{\min }\right)+w\left(\frac{N K}{\sqrt{p(k)}}-\frac{C d}{M}\right)<q_{\min }
$$

and

$$
(1-w)\left(\frac{q_{\max }-q_{\min }}{p_{\max }} p(k)+q_{\min }\right)+w\left(\frac{N K}{\sqrt{p(k)}}-\frac{C d}{M}\right) \geq 0 .
$$

Hence we have

$$
0 \leq(1-w)\left(\frac{q_{\max }-q_{\min }}{p_{\max }} p(k)+q_{\min }\right)+w\left(\frac{N K}{\sqrt{p(k)}}-\frac{C d}{M}\right)<q_{\min },
$$


International Journal of Bifurcation and Chaos

$0 \leq(1-w) \bar{q}(k)+w\left(\frac{N K}{\sqrt{\frac{\bar{q}(k)-q_{\min }}{q_{\max }-q_{\min }} p_{\max }}}-\frac{C d}{M}\right)<q_{\min }$ and $0 \leq \bar{q}(k+1)<q_{\min }$. Consequently, we have $p(k+1)=0$. This completes the proof.

A.5 Dynamics of the average queue size in the fifth subset

Denote

$\begin{array}{ll}P_{5} & \equiv\left\{p(k): \frac{(1-w)\left(q_{\max }-q_{\min }\right)}{p_{\max }}(p(k))^{\frac{3}{2}}+w N K+\left((1-w) q_{\min }-\frac{w C d}{M}-q_{\max }\right)(p(k))^{\frac{1}{2}} \geq 0\right\} \quad \text { and } \\ Q_{5} & \equiv\left\{\bar{q}(k): \bar{q}(k)=\frac{q_{\max }-q_{\min }}{p_{\max }} p(k)+q_{\min } \text { and } p(k) \in P_{5}\right\} \text {. If } \quad q_{\min } \leq \bar{q}(k)<q_{\max } \text {, }\end{array}$ $\bar{q}(k) \leq \frac{q_{\max }-q_{\min }}{p_{\max }}\left(\frac{M N K}{C d}\right)^{2}+q_{\min } \quad$ and $\quad \bar{q}(k) \in Q_{5}, \quad$ then $\quad p(k)=\frac{\bar{q}(k)-q_{\min }}{q_{\max }-q_{\min }} p_{\max }$, $\bar{q}(k+1)=(1-w) \bar{q}(k)+w\left(\frac{N K}{\sqrt{\frac{\bar{q}(k)-q_{\min }}{q_{\max }-q_{\min }} p_{\max }}}-\frac{C d}{M}\right)$ and $p(k+1)=1$. Denote $\quad s_{5}(k)=1$ and $s_{j}(k)=0$ for $j \neq 5$.

Proof:

It is obvious to see that if $q_{\min } \leq \bar{q}(k)<q_{\max }$, then $p(k)=\frac{\bar{q}(k)-q_{\min }}{q_{\max }-q_{\min }} p_{\max }$. Since $\bar{q}(k) \leq \frac{q_{\max }-q_{\min }}{p_{\max }}\left(\frac{M N K}{C d}\right)^{2}+q_{\min }$, this implies that $\frac{\bar{q}(k)-q_{\min }}{q_{\max }-q_{\min }} p_{\max } \leq\left(\frac{M N K}{C d}\right)^{2}, \quad p(k) \leq\left(\frac{M N K}{C d}\right)^{2}$, $\frac{C d}{K} \leq \frac{M N}{\sqrt{p(k)}}$ and $\frac{M N}{\sqrt{p(k)}}-\frac{C d}{K} \geq 0$. Hence, we have

$$
\bar{q}(k+1)=(1-w) \bar{q}(k)+w\left(\frac{N K}{\sqrt{\frac{\bar{q}(k)-q_{\min }}{q_{\max }-q_{\min }}} p_{\max }}-\frac{C d}{M}\right) .
$$


International Journal of Bifurcation and Chaos

As $\bar{q}(k) \in Q_{5}$, we have $p(k) \in P_{5}$. In other words, we have

$$
\frac{(1-w)\left(q_{\max }-q_{\min }\right)}{p_{\max }}(p(k))^{\frac{3}{2}}+w N K+\left((1-w) q_{\min }-\frac{w C d}{M}-q_{\max }\right)(p(k))^{\frac{1}{2}} \geq 0 .
$$

This implies that

$$
\begin{gathered}
\frac{(1-w)\left(q_{\max }-q_{\min }\right)}{p_{\max }} p(k)+\frac{w N K}{\sqrt{p(k)}}+(1-w) q_{\min }-\frac{w C d}{M}-q_{\max } \geq 0, \\
(1-w)\left(\frac{q_{\max }-q_{\min }}{p_{\max }} p(k)+q_{\min }\right)+w\left(\frac{N K}{\sqrt{p(k)}}-\frac{C d}{M}\right) \geq q_{\max },
\end{gathered}
$$

$(1-w) \bar{q}(k)+w\left(\frac{N K}{\sqrt{\frac{\bar{q}(k)-q_{\min }}{q_{\max }-q_{\min }} p_{\max }}}-\frac{C d}{M}\right) \geq q_{\max }$ and $\bar{q}(k+1) \geq q_{\max }$. Hence, we have $p(k+1)=1$.

This completes the proof.

A.6 Dynamics of the average queue size in the sixth subset

$$
\text { Denote } \quad P_{6} \equiv\left\{\begin{array}{l}
p(k): \frac{(1-w)\left(q_{\max }-q_{\min }\right)}{p_{\max }}(p(k))^{\frac{3}{2}}+w N K+\left((1-w) q_{\min }-\frac{w C d}{M}-q_{\min }\right)(p(k))^{\frac{1}{2}} \geq 0 \\
\text { and } \frac{(1-w)\left(q_{\max }-q_{\min }\right)}{p_{\max }}(p(k))^{\frac{3}{2}}+w N K+\left((1-w) q_{\min }-\frac{w C d}{M}-q_{\max }\right)(p(k))^{\frac{1}{2}}<0
\end{array}\right\}
$$

and $\quad Q_{6} \equiv\left\{\bar{q}(k): \bar{q}(k)=\frac{q_{\max }-q_{\min }}{p_{\max }} p(k)+q_{\min }\right.$ and $\left.p(k) \in P_{6}\right\} \quad$. If $\quad q_{\min } \leq \bar{q}(k)<q_{\max }$,

$$
\bar{q}(k) \leq \frac{q_{\max }-q_{\min }}{p_{\max }}\left(\frac{M N K}{C d}\right)^{2}+q_{\min } \quad \text { and } \quad \bar{q}(k) \in Q_{6} \quad, \quad \text { then } \quad p(k)=\frac{\bar{q}(k)-q_{\min }}{q_{\max }-q_{\min }} p_{\max },
$$

$\bar{q}(k+1)=(1-w) \bar{q}(k)+w\left(\frac{N K}{\sqrt{\frac{\bar{q}(k)-q_{\min }}{q_{\max }-q_{\min }} p_{\max }}}-\frac{C d}{M}\right)$ and 
International Journal of Bifurcation and Chaos

$$
p(k+1)=\frac{(1-w) \bar{q}(k)+w\left(\frac{N K}{\sqrt{\frac{\bar{q}(k)-q_{\min }}{q_{\max }-q_{\min }} p_{\max }}}-\frac{C d}{M}\right)-q_{\min }}{q_{\max }-q_{\min }} p_{\max } .
$$

Denote $s_{6}(k)=1$ and $s_{j}(k)=0$ for $j \neq 6$.

Proof:

It is obvious to see that if $q_{\min } \leq \bar{q}(k)<q_{\max }$, then $p(k)=\frac{\bar{q}(k)-q_{\min }}{q_{\max }-q_{\min }} p_{\max }$. Since $\bar{q}(k) \leq \frac{q_{\max }-q_{\min }}{p_{\max }}\left(\frac{M N K}{C d}\right)^{2}+q_{\min }$, this implies that $\frac{\bar{q}(k)-q_{\min }}{q_{\max }-q_{\min }} p_{\max } \leq\left(\frac{M N K}{C d}\right)^{2}, \quad p(k) \leq\left(\frac{M N K}{C d}\right)^{2}$, $\frac{C d}{K} \leq \frac{M N}{\sqrt{p(k)}}$ and $\frac{M N}{\sqrt{p(k)}}-\frac{C d}{K} \geq 0$. Hence, we have

$$
\bar{q}(k+1)=(1-w) \bar{q}(k)+w\left(\frac{N K}{\sqrt{\frac{\bar{q}(k)-q_{\min }}{q_{\max }-q_{\min }}} p_{\max }}-\frac{C d}{M}\right) .
$$

As $\bar{q}(k) \in Q_{6}$, we have $p(k) \in P_{6}$. In other words, we have

$$
\frac{(1-w)\left(q_{\max }-q_{\min }\right)}{p_{\max }}(p(k))^{\frac{3}{2}}+w N K+\left((1-w) q_{\min }-\frac{w C d}{M}-q_{\min }\right)(p(k))^{\frac{1}{2}} \geq 0
$$

and

$$
\frac{(1-w)\left(q_{\max }-q_{\min }\right)}{p_{\max }}(p(k))^{\frac{3}{2}}+w N K+\left((1-w) q_{\min }-\frac{w C d}{M}-q_{\max }\right)(p(k))^{\frac{1}{2}}<0 .
$$

This implies that

$$
\frac{(1-w)\left(q_{\max }-q_{\min }\right)}{p_{\max }} p(k)+\frac{w N K}{\sqrt{p(k)}}+(1-w) q_{\min }-\frac{w C d}{M}-q_{\min } \geq 0
$$

and

$$
\frac{(1-w)\left(q_{\max }-q_{\min }\right)}{p_{\max }} p(k)+\frac{w N K}{\sqrt{p(k)}}+(1-w) q_{\min }-\frac{w C d}{M}-q_{\max }<0,
$$

as well as 
International Journal of Bifurcation and Chaos

$$
(1-w)\left(\frac{q_{\max }-q_{\min }}{p_{\max }} p(k)+q_{\min }\right)+w\left(\frac{N K}{\sqrt{p(k)}}-\frac{C d}{M}\right) \geq q_{\min }
$$

and

$$
(1-w)\left(\frac{q_{\max }-q_{\min }}{p_{\max }} p(k)+q_{\min }\right)+w\left(\frac{N K}{\sqrt{p(k)}}-\frac{C d}{M}\right)<q_{\max }
$$

Hence, we have

$$
q_{\min } \leq(1-w)\left(\frac{q_{\max }-q_{\min }}{p_{\max }} p(k)+q_{\min }\right)+w\left(\frac{N K}{\sqrt{p(k)}}-\frac{C d}{M}\right)<q_{\max }
$$

$q_{\min } \leq(1-w) \bar{q}(k)+w\left(\frac{N K}{\sqrt{\frac{\bar{q}(k)-q_{\min }}{q_{\max }-q_{\min }} p_{\max }}}-\frac{C d}{M}\right)<q_{\max }$ and $q_{\min } \leq \bar{q}(k+1)<q_{\max }$. Consequently, we

have $p(k+1)=\frac{\bar{q}(k+1)-q_{\min }}{q_{\text {max }}-q_{\text {min }}} p_{\max }=\frac{(1-w) \bar{q}(k)+w\left(\frac{N K}{\left.\sqrt{\frac{\bar{q}(k)-q_{\min }}{q_{\max }-q_{\min }}}-\frac{C d}{M}\right)-q_{\min }}\right.}{q_{\text {max }}-q_{\min }} p_{\max } \cdot$ This completes the proof.

A.7 Dynamics of the average queue size in the seventh subset

If $\quad q_{\min } \leq \bar{q}(k)<q_{\max }, \quad \bar{q}(k)>\left(\frac{M N K}{C d}\right)^{2} \frac{q_{\max }-q_{\min }}{p_{\max }}+q_{\min } \quad$ and $\quad 0 \leq \bar{q}(k)<\frac{q_{\min }}{1-w}, \quad$ then $p(k)=\frac{\bar{q}(k)-q_{\min }}{q_{\max }-q_{\min }} p_{\max }, \quad \bar{q}(k+1)=(1-w) \bar{q}(k)$ and $p(k+1)=0$. Denote $s_{7}(k)=1$ and $s_{j}(k)=0$ for $j \neq 7$.

Proof:

It is obvious to see that if $q_{\min } \leq \bar{q}(k)<q_{\max }$, then $p(k)=\frac{\bar{q}(k)-q_{\min }}{q_{\max }-q_{\min }} p_{\max }$. Since $\bar{q}(k)>\left(\frac{M N K}{C d}\right)^{2} \frac{q_{\max }-q_{\min }}{p_{\max }}+q_{\min }$, this implies that $\frac{\bar{q}(k)-q_{\min }}{q_{\max }-q_{\min }} p_{\max }>\left(\frac{M N K}{C d}\right)^{2}, \quad p(k)>\left(\frac{M N K}{C d}\right)^{2}$, 
International Journal of Bifurcation and Chaos

$\frac{C d}{K}>\frac{M N}{\sqrt{p(k)}}$ and $\frac{M N}{\sqrt{p(k)}}-\frac{C d}{K}<0$. Hence, we have $\bar{q}(k+1)=(1-w) \bar{q}(k)$. As $0 \leq \bar{q}(k)<\frac{q_{\min }}{1-w}$, we have $0 \leq(1-w) \bar{q}(k)<q_{\min }, \quad 0 \leq \bar{q}(k+1)<q_{\min }$ and $p(k+1)=0$. This completes the proof.

A.8 Dynamics of the average queue size in the eighth subset

If $\quad q_{\min } \leq \bar{q}(k)<q_{\max }, \quad \bar{q}(k)>\left(\frac{M N K}{C d}\right)^{2} \frac{q_{\max }-q_{\min }}{p_{\max }}+q_{\min } \quad$ and $\quad \bar{q}(k) \geq \frac{q_{\max }}{1-w} \quad, \quad$ then $p(k)=\frac{\bar{q}(k)-q_{\min }}{q_{\max }-q_{\min }} p_{\max }, \quad \bar{q}(k+1)=(1-w) \bar{q}(k)$ and $p(k+1)=1$. Denote $s_{8}(k)=1$ and $s_{j}(k)=0$ for $j \neq 8$.

Proof:

It is obvious to see that if $q_{\min } \leq \bar{q}(k)<q_{\max }$, then $p(k)=\frac{\bar{q}(k)-q_{\min }}{q_{\max }-q_{\min }} p_{\max }$. Since $\bar{q}(k)>\left(\frac{M N K}{C d}\right)^{2} \frac{q_{\max }-q_{\min }}{p_{\max }}+q_{\min }$, this implies that $\frac{\bar{q}(k)-q_{\min }}{q_{\max }-q_{\min }} p_{\max }>\left(\frac{M N K}{C d}\right)^{2}, p(k)>\left(\frac{M N K}{C d}\right)^{2}$, $\frac{C d}{K}>\frac{M N}{\sqrt{p(k)}}$ and $\frac{M N}{\sqrt{p(k)}}-\frac{C d}{K}<0$. Hence, we have $\bar{q}(k+1)=(1-w) \bar{q}(k)$. As $\bar{q}(k) \geq \frac{q_{\max }}{1-w}$, we have $(1-w) \bar{q}(k) \geq q_{\max }, \quad \bar{q}(k+1) \geq q_{\max }$ and $p(k+1)=1$. This completes the proof.

A.9 Dynamics of the average queue size in the ninth subset

If $q_{\min } \leq \bar{q}(k)<q_{\max }, \quad \bar{q}(k)>\left(\frac{M N K}{C d}\right)^{2} \frac{q_{\max }-q_{\min }}{p_{\max }}+q_{\min } \quad$ and $\quad \frac{q_{\max }}{1-w}>\bar{q}(k) \geq \frac{q_{\min }}{1-w}$, then $p(k)=\frac{\bar{q}(k)-q_{\min }}{q_{\max }-q_{\min }} p_{\max }, \quad \bar{q}(k+1)=(1-w) \bar{q}(k) \quad$ and $\quad p(k+1)=\frac{(1-w) \bar{q}(k)-q_{\min }}{q_{\max }-q_{\min }} p_{\max } . \quad$ Denote $s_{9}(k)=1$ and $s_{j}(k)=0$ for $j \neq 9$.

Proof:

It is obvious to see that if $q_{\min } \leq \bar{q}(k)<q_{\max }$, then $p(k)=\frac{\bar{q}(k)-q_{\min }}{q_{\max }-q_{\min }} p_{\max }$. Since $\bar{q}(k)>\left(\frac{M N K}{C d}\right)^{2} \frac{q_{\max }-q_{\min }}{p_{\max }}+q_{\min }$, this implies that $\frac{\bar{q}(k)-q_{\min }}{q_{\max }-q_{\min }} p_{\max }>\left(\frac{M N K}{C d}\right)^{2}, p(k)>\left(\frac{M N K}{C d}\right)^{2}$, $\frac{C d}{K}>\frac{M N}{\sqrt{p(k)}} \quad$ and $\quad \frac{M N}{\sqrt{p(k)}}-\frac{C d}{K}<0 \quad$. Hence, we have $\quad \bar{q}(k+1)=(1-w) \bar{q}(k)$. 
International Journal of Bifurcation and Chaos

$\frac{q_{\max }}{1-w}>\bar{q}(k) \geq \frac{q_{\min }}{1-w} \quad$, we have $\quad q_{\max }>(1-w) \bar{q}(k) \geq q_{\min } \quad, \quad q_{\max }>\bar{q}(k+1) \geq q_{\min } \quad$ and $p(k+1)=\frac{\bar{q}(k+1)-q_{\min }}{q_{\max }-q_{\min }} p_{\max }=\frac{(1-w) \bar{q}(k)-q_{\min }}{q_{\max }-q_{\min }} p_{\max }$. This completes the proof.

A.10 Dynamics of the average queue size in the tenth subset

If $\bar{q}(k) \geq q_{\max }, 1 \leq \frac{M N K}{C d}$ and $\frac{w\left(\frac{C d}{M}-N K\right)}{1-w} \leq \bar{q}(k)<\frac{q_{\min }-w\left(N K-\frac{C d}{M}\right)}{1-w}$, then $p(k)=1$, $\bar{q}(k+1)=(1-w) \bar{q}(k)+w\left(N K-\frac{C d}{M}\right)$ and $\quad p(k+1)=0$. Denote $s_{10}(k)=1$ and $s_{j}(k)=0$ for $j \neq 10$.

Proof:

It is obvious to see that if $\bar{q}(k) \geq q_{\max }$, then $p(k)=1$. Since $\sqrt{p(k)}=1 \leq \frac{M N K}{C d}$, this implies that $\frac{C d}{K} \leq \frac{M N}{\sqrt{p(k)}}$ and $\frac{M N}{\sqrt{p(k)}}-\frac{C d}{K} \geq 0$. Hence, we have $\bar{q}(k+1)=(1-w) \bar{q}(k)+w\left(N K-\frac{C d}{M}\right)$. As $\frac{w\left(\frac{C d}{M}-N K\right)}{1-w} \leq \bar{q}(k)<\frac{q_{\min }-w\left(N K-\frac{C d}{M}\right)}{1-w}$, we have $0 \leq(1-w) \bar{q}(k)+w\left(N K-\frac{C d}{M}\right)<q_{\min }$, $0 \leq \bar{q}(k+1)<q_{\min }$ and $p(k+1)=0$. This completes the proof.

\section{A.11 Dynamics of the average queue size in the eleventh subset}

If $\quad \bar{q}(k) \geq q_{\max } \quad, \quad 1 \leq \frac{M N K}{C d}$ and $\quad \bar{q}(k) \geq \frac{q_{\max }-w\left(N K-\frac{C d}{M}\right)}{1-w}$, then $\quad p(k)=1$, $\bar{q}(k+1)=(1-w) \bar{q}(k)+w\left(N K-\frac{C d}{M}\right)$ and $\quad p(k+1)=1$. Denote $s_{11}(k)=1$ and $s_{j}(k)=0$ for $j \neq 11$.

Proof:

It is obvious to see that if $\bar{q}(k) \geq q_{\max }$, then $p(k)=1$. Since $\sqrt{p(k)}=1 \leq \frac{M N K}{C d}$, this implies that $\frac{C d}{K} \leq \frac{M N}{\sqrt{p(k)}}$ and $\frac{M N}{\sqrt{p(k)}}-\frac{C d}{K} \geq 0$. Hence, we have $\bar{q}(k+1)=(1-w) \bar{q}(k)+w\left(N K-\frac{C d}{M}\right)$. As $\bar{q}(k) \geq \frac{q_{\max }-w\left(N K-\frac{C d}{M}\right)}{1-w}$, we have $(1-w) \bar{q}(k)+w\left(N K-\frac{C d}{M}\right) \geq q_{\max }, \quad \bar{q}(k+1) \geq q_{\max }$ and 
International Journal of Bifurcation and Chaos

$p(k+1)=1$. This completes the proof.

A.12 Dynamics of the average queue size in the twelfth subset

$$
\begin{gathered}
\text { If } \bar{q}(k) \geq q_{\max }, \quad 1 \leq \frac{M N K}{C d} \text { and } \frac{q_{\max }-w\left(N K-\frac{C d}{M}\right)}{1-w}>\bar{q}(k) \geq \frac{q_{\min }-w\left(N K-\frac{C d}{M}\right)}{1-w} \text {, then } \\
p(k)=1, \quad \bar{q}(k+1)=(1-w) \bar{q}(k)+w\left(N K-\frac{C d}{M}\right) \text { and } \\
p(k+1)=\frac{(1-w) \bar{q}(k)+w\left(N K-\frac{C d}{M}\right)-q_{\min }}{q_{\max }-q_{\min }} p_{\max } .
\end{gathered}
$$

Denote $s_{12}(k)=1$ and $s_{j}(k)=0$ for $j \neq 12$.

Proof:

It is obvious to see that if $\bar{q}(k) \geq q_{\max }$, then $p(k)=1$. Since $\sqrt{p(k)}=1 \leq \frac{M N K}{C d}$, this implies that $\frac{C d}{K} \leq \frac{M N}{\sqrt{p(k)}}$ and $\frac{M N}{\sqrt{p(k)}}-\frac{C d}{K} \geq 0$. Hence, we have $\bar{q}(k+1)=(1-w) \bar{q}(k)+w\left(N K-\frac{C d}{M}\right)$. As $\frac{q_{\max }-w\left(N K-\frac{C d}{M}\right)}{1-w}>\bar{q}(k) \geq \frac{q_{\min }-w\left(N K-\frac{C d}{M}\right)}{1-w}$, we have

$$
q_{\max }>(1-w) \bar{q}(k)+w\left(N K-\frac{C d}{M}\right) \geq q_{\min },
$$

$q_{\max }>\bar{q}(k+1) \geq q_{\min } \quad$ and $\quad p(k+1)=\frac{\bar{q}(k+1)-q_{\min }}{q_{\max }-q_{\min }} p_{\max }=\frac{(1-w) \bar{q}(k)+w\left(N K-\frac{C d}{M}\right)-q_{\min }}{q_{\max }-q_{\min }} p_{\max }$.

This completes the proof.

A.13 Dynamics of the average queue size in the thirteenth subset

If $\bar{q}(k) \geq q_{\max }, \frac{M N K}{C d}<1$ and $0 \leq \bar{q}(k)<\frac{q_{\min }}{1-w}$, then $p(k)=1, \bar{q}(k+1)=(1-w) \bar{q}(k)$ and $p(k+1)=0$. Denote $s_{13}(k)=1$ and $s_{j}(k)=0$ for $j \neq 13$.

Proof:

It is obvious to see that if $\bar{q}(k) \geq q_{\max }$, then $p(k)=1$. Since $\frac{M N K}{C d}<1=\sqrt{p(k)}$, this implies that $\frac{C d}{K}>\frac{M N}{\sqrt{p(k)}}$ and $\frac{M N}{\sqrt{p(k)}}-\frac{C d}{K}<0$. Hence, we have $\bar{q}(k+1)=(1-w) \bar{q}(k)$. As 
International Journal of Bifurcation and Chaos

$0 \leq \bar{q}(k)<\frac{q_{\min }}{1-w}$, we have $0 \leq(1-w) \bar{q}(k)<q_{\min }, \quad 0 \leq \bar{q}(k+1)<q_{\min }$ and $\quad p(k+1)=0$. This completes the proof.

A.14 Dynamics of the average queue size in the fourteenth subset

If $\bar{q}(k) \geq q_{\max }, \frac{M N K}{C d}<1$ and $\bar{q}(k) \geq \frac{q_{\max }}{1-w}$, then $p(k)=1, \quad \bar{q}(k+1)=(1-w) \bar{q}(k)$ and $p(k+1)=1$. Denote $s_{14}(k)=1$ and $s_{j}(k)=0$ for $j \neq 14$.

Proof:

It is obvious to see that if $\bar{q}(k) \geq q_{\max }$, then $p(k)=1$. Since $\frac{M N K}{C d}<1=\sqrt{p(k)}$, this implies that $\frac{C d}{K}>\frac{M N}{\sqrt{p(k)}}$ and $\frac{M N}{\sqrt{p(k)}}-\frac{C d}{K}<0$. Hence, we have $\bar{q}(k+1)=(1-w) \bar{q}(k)$. As $\bar{q}(k) \geq \frac{q_{\max }}{1-w}$, we have $(1-w) \bar{q}(k) \geq q_{\max }, \quad \bar{q}(k+1) \geq q_{\max }$ and $p(k+1)=1$. This completes the proof.

A.15 Dynamics of the average queue size in the fifteenth subset

If $\bar{q}(k) \geq q_{\max }, \frac{M N K}{C d}<1$ and $\frac{q_{\max }}{1-w}>\bar{q}(k) \geq \frac{q_{\min }}{1-w}$, then $p(k)=1, \quad \bar{q}(k+1)=(1-w) \bar{q}(k)$ and $p(k+1)=\frac{(1-w) \bar{q}(k)-q_{\min }}{q_{\max }-q_{\min }} p_{\max }$. Denote $s_{15}(k)=1$ and $s_{j}(k)=0$ for $j \neq 15$.

Proof:

It is obvious to see that if $\bar{q}(k) \geq q_{\max }$, then $p(k)=1$. Since $\frac{M N K}{C d}<1=\sqrt{p(k)}$, this implies that $\frac{C d}{K}>\frac{M N}{\sqrt{p(k)}}$ and $\frac{M N}{\sqrt{p(k)}}-\frac{C d}{K}<0$. Hence, we have $\bar{q}(k+1)=(1-w) \bar{q}(k)$. As $\frac{q_{\max }}{1-w}>\bar{q}(k) \geq \frac{q_{\min }}{1-w} \quad$, we have $\quad q_{\max }>(1-w) \bar{q}(k) \geq q_{\min } \quad, \quad q_{\max }>\bar{q}(k+1) \geq q_{\min } \quad$ and $p(k+1)=\frac{\bar{q}(k+1)-q_{\min }}{q_{\max }-q_{\min }} p_{\max }=\frac{(1-w) \bar{q}(k)-q_{\min }}{q_{\max }-q_{\min }} p_{\max }$. This completes the proof.

$\bar{q}(k)$ could be switched among these fifteen subsets according to the value of $\mathbf{s}(k)$. Denote

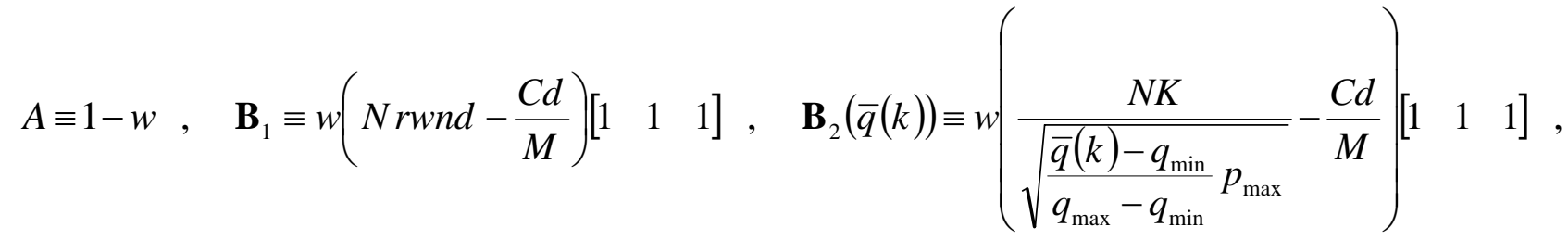


International Journal of Bifurcation and Chaos

$$
\begin{array}{r}
\mathbf{B}_{3} \equiv\left[\begin{array}{lll}
0 & 0 & 0
\end{array}\right], \quad \mathbf{B}_{4} \equiv w\left(N K-\frac{C d}{M}\right)\left[\begin{array}{lll}
1 & 1 & 1
\end{array}\right], \quad \mathbf{B}_{5} \equiv\left[\begin{array}{lll}
0 & 0 & 0
\end{array}\right] \text { and } \\
\mathbf{B}^{\prime}(\bar{q}(k)) \equiv\left[\begin{array}{lllll}
\mathbf{B}_{1} & \mathbf{B}_{2}(\bar{q}(k)) & \mathbf{B}_{3} & \mathbf{B}_{4} & \mathbf{B}_{5}
\end{array}\right],
\end{array}
$$

then the dynamics of the averages queue size of the RED algorithm can be represented by $\bar{q}(k+1)=A \bar{q}(k)+u(k)$, where $u(k)=\mathbf{B}^{\prime}(\bar{q}(k)) \mathbf{s}(k)$. This model can be represented via a closed loop feedback system having a linear time-invariant plant with the four state space constants $A, 1,1$ and 0 , and a positive nonlinear feedback system with its input-output relationship governed by $u(k)=\mathbf{B}^{\prime}(\bar{q}(k)) \mathbf{s}(k)$.

This proposed symbolic dynamical model is useful for designing both the system parameters and the initial conditions so that the average queue size of the RED algorithm would converge to a fixed point. Also, the boundedness of the average queue size of the RED algorithm could be determined easily via the proposed symbolic dynamical model. As $0<w<1, A$ is strictly stable. If both the system parameters and the initial conditions is designed so that $u(k)$ is bounded, then $\bar{q}(k)$ is guaranteed to be bounded.

It is worth noting that not all subsets contain a fixed point. The conditions on both the system parameters and the initial conditions that the average queue size of the RED algorithm would converge to a fixed point are derived in Section B as follows.

\section{B. Conditions for exhibiting fixed point behaviors}

Since the average queue size of the RED algorithm is required to converge to a fixed point, it is important to characterize the conditions on both the system parameters and the initial conditions so that the average queue size of the RED algorithm would converge to a fixed point. These conditions are summarized in the following lemmas:

\section{Lemma 1}

The fixed point of the average queue size of the RED algorithm would not be located at $S_{2} \cup S_{3} \cup S_{4} \cup S_{5} \cup S_{7} \cup S_{8} \cup S_{9} \cup S_{10} \cup S_{12} \cup S_{13} \cup S_{14} \cup S_{15}$

Proof: 
International Journal of Bifurcation and Chaos

As $\quad p(k) \neq p(k+1)$ for $\bar{q}(k) \in S_{2} \cup S_{3} \cup S_{4} \cup S_{5} \cup S_{7} \cup S_{8} \cup S_{10} \cup S_{12} \cup S_{13} \cup S_{15}$, the fixed point of the average queue size of the RED algorithm could not be located at $S_{2} \cup S_{3} \cup S_{4} \cup S_{5} \cup S_{7} \cup S_{8} \cup S_{10} \cup S_{12} \cup S_{13} \cup S_{15}$. As the dynamics of the average queue size of the RED algorithm for $\bar{q}(k) \in S_{14}$ is governed by $\bar{q}(k+1)=(1-w) \bar{q}(k)$, if the fixed point is located at $S_{14}$, then the fixed point has to be located at the origin, but it contradicts to $\bar{q}(k) \geq q_{\max }$. Hence, the fixed point of the average queue size of the RED algorithm could not be located at $S_{14}$. Similarly, the fixed point of the average queue size of the RED algorithm could not be located at $S_{9}$. This completed the proof.

Although the dynamics of the average queue size for $\bar{q}(k) \in S_{1} \cup S_{6} \cup S_{11}$ is characterized, it is not guaranteed that $\bar{q}(k+1) \in S_{1}$ for $\bar{q}(k) \in S_{1}, \bar{q}(k+1) \in S_{6}$ for $\bar{q}(k) \in S_{6}$ and $\bar{q}(k+1) \in S_{11}$ for $\bar{q}(k) \in S_{11}$. Further conditions are required to be imposed and the details are discussed in the following lemmas.

\section{Lemma 2}

If $\exists k_{0} \geq 0$ such that

i) $N r w n d-\frac{C d}{M}<q_{\min }$,

ii) $w \leq \frac{1}{2}$ and Nrwnd $-\frac{C d}{M} \geq 0$

and

iii) $s_{1}\left(k_{0}\right)=1$,

then

i) $s_{1}(k)=1 \quad \forall k \geq k_{0}$,

ii) $p(k)=0 \quad \forall k \geq k_{0}$

and 
International Journal of Bifurcation and Chaos

iii) $\bar{q}(k) \rightarrow N r w n d-\frac{C d}{M}$ as $k \rightarrow+\infty$.

Proof:

As $s_{1}\left(k_{0}\right)=1$, this implies that $0 \leq \bar{q}\left(k_{0}+1\right)<q_{\min }$. Since $w \leq \frac{1}{2}$ and $N r w n d-\frac{C d}{M} \geq 0$, we have $\quad(2 w-1)\left(N r w n d-\frac{C d}{M}\right) \leq 0 \quad, \quad w\left(N r w n d-\frac{C d}{M}\right) \leq(1-w)\left(N r w n d-\frac{C d}{M}\right) \quad$ and $\frac{w\left(N r w n d-\frac{C d}{M}\right)}{1-w} \leq N r w n d-\frac{C d}{M}$. On the other hand, as $N r w n d-\frac{C d}{M}<q_{\min }$, we have $N r w n d-\frac{C d}{M}-w\left(N r w n d-\frac{C d}{M}\right)<q_{\min }-w\left(N r w n d-\frac{C d}{M}\right)$

$(1-w)\left(N r w n d-\frac{C d}{M}\right)<q_{\min }-w\left(N r w n d-\frac{C d}{M}\right), \quad N r w n d-\frac{C d}{M}<\frac{q_{\min }-w\left(N r w n d-\frac{C d}{M}\right)}{1-w}$ and $\frac{w\left(N r w n d-\frac{C d}{M}\right)}{1-w} \leq N r w n d-\frac{C d}{M}<\frac{q_{\min }-w\left(N r w n d-\frac{C d}{M}\right)}{1-w}$. Since $s_{1}\left(k_{0}\right)=1$, we have $\frac{w\left(\frac{C d}{M}-N r w n d\right)}{1-w} \leq \bar{q}\left(k_{0}\right)<\frac{q_{\min }-w\left(N r w n d-\frac{C d}{M}\right)}{1-w}$. As $\bar{q}\left(k_{0}+1\right)$ is a convex combinational of $\bar{q}\left(k_{0}\right)$ and Nrwnd $-\frac{C d}{M}$, we have $\frac{w\left(N r w n d-\frac{C d}{M}\right)}{1-w} \leq \bar{q}\left(k_{0}+1\right)<\frac{q_{\min }-w\left(N r w n d-\frac{C d}{M}\right)}{1-w}$. This implies that $\bar{q}\left(k_{0}+1\right) \in S_{1}$. Similarly, we have $\bar{q}(k) \in S_{1} \quad \forall k \geq k_{0}$. As

$$
\bar{q}\left(k_{0}+k\right)=(1-w)^{k}\left(\bar{q}\left(k_{0}\right)-N r w n d+\frac{C d}{M}\right)+N r w n d-\frac{C d}{M} \quad \forall k \geq 0,
$$

we have $\bar{q}(k) \rightarrow N r w n d-\frac{C d}{M}$ as $k \rightarrow+\infty$. This completes the proof.

This lemma characterizes the condition on both the system parameters and the initial conditions that the average queue size of the RED algorithm would converge to a fixed point in the first subset. It is worth noting that if the second condition in Lemma 2 is changed to $w \geq \frac{1}{2}$ and Nrwnd $-\frac{C d}{M} \leq 0$, the same result would been obtained. However, due to the physical nature of $\bar{q}(k), \quad \bar{q}(k) \geq 0 \quad \forall k \geq 0$. Since $\bar{q}(k) \rightarrow N r w n d-\frac{C d}{M}$ as $k \rightarrow+\infty$, the physical nature of $\bar{q}(k)$ is 
International Journal of Bifurcation and Chaos

violated. Hence, this case has not been considered in Lemma 2.

\section{Lemma 3}

If $\exists k_{0} \geq 0$ such that

i) $N K-\frac{C d}{M} \geq q_{\max }$

and

ii) $s_{11}\left(k_{0}\right)=1$,

then

i) $s_{11}(k)=1 \quad \forall k \geq k_{0}$,

ii) $p(k)=1 \quad \forall k \geq k_{0}$

and

iii) $\bar{q}(k) \rightarrow N K-\frac{C d}{M}$ as $k \rightarrow+\infty$.

Proof:

As $\quad s_{11}\left(k_{0}\right)=1$, this implies that $\bar{q}\left(k_{0}+1\right) \geq q_{\max }$. Since $N K-\frac{C d}{M} \geq q_{\max }$, we have $N K-\frac{C d}{M}-w\left(N K-\frac{C d}{M}\right) \geq q_{\max }-w\left(N K-\frac{C d}{M}\right), \quad(1-w)\left(N K-\frac{C d}{M}\right) \geq q_{\max }-w\left(N K-\frac{C d}{M}\right) \quad$ and $N K-\frac{C d}{M} \geq \frac{q_{\max }-w\left(N K-\frac{C d}{M}\right)}{1-w}$. As $s_{11}\left(k_{0}\right)=1$, we have $\bar{q}\left(k_{0}\right) \geq \frac{q_{\max }-w\left(N K-\frac{C d}{M}\right)}{1-w}$. Since $\bar{q}\left(k_{0}+1\right) \quad$ is a convex combinational of $\bar{q}\left(k_{0}\right)$ and $N K-\frac{C d}{M}$, we have $\bar{q}\left(k_{0}+1\right) \geq \frac{q_{\max }-w\left(N K-\frac{C d}{M}\right)}{1-w}$. This implies that $\bar{q}\left(k_{0}+1\right) \in S_{11}$. Similarly, we have $\bar{q}(k) \in S_{11}$ $\forall k \geq k_{0} . \quad$ As $\quad \bar{q}\left(k_{0}+k\right)=(1-w)^{k}\left(\bar{q}\left(k_{0}\right)-N K+\frac{C d}{M}\right)+N K-\frac{C d}{M} \quad \forall k \geq 0 \quad$, we have $\bar{q}(k) \rightarrow N K-\frac{C d}{M}$ as $k \rightarrow+\infty$. This completes the proof.

This lemma characterizes the condition on both the system parameters and the initial conditions that the average queue size of the RED algorithm would converge to a fixed point in the 
International Journal of Bifurcation and Chaos

eleventh subset.

\section{Lemma 4}

If $\exists k_{0} \geq 0$ such that

i) $s_{6}\left(k_{0}\right)=1$,

ii) $(1-w) \bar{q}\left(k_{0}\right)+w\left(\frac{N K}{\sqrt{\frac{\bar{q}\left(k_{0}\right)-q_{\min }}{q_{\max }-q_{\min }} p_{\max }}}-\frac{C d}{M}\right)>q_{\min }+\frac{q_{\max }-q_{\min }}{p_{\max }}\left(\frac{N K}{q_{\max }+\frac{C d}{M}}\right)^{2}$,

iii) $(1-w) \bar{q}\left(k_{0}\right)+w\left(\frac{N K}{\sqrt{\frac{\bar{q}\left(k_{0}\right)-q_{\min }}{q_{\max }-q_{\min }} p_{\max }}}-\frac{C d}{M}\right) \leq q_{\min }+\frac{q_{\max }-q_{\min }}{p_{\max }}\left(\frac{N K}{q_{\min }+\frac{C d}{M}}\right)^{2}$

and

iv) $(1-w) \bar{q}\left(k_{0}\right)+w\left(\frac{N K}{\sqrt{\frac{\bar{q}\left(k_{0}\right)-q_{\min }}{q_{\max }-q_{\min }} p_{\max }}}-\frac{C d}{M}\right) \leq q_{\min }+\frac{q_{\max }-q_{\min }}{p_{\max }}\left(\frac{M N K}{C d}\right)^{2}$,

then

$s_{6}(k)=1 \quad \forall k \geq k_{0}$.

Proof:

As $s_{6}\left(k_{0}\right)=1$, we have $q_{\max }>\bar{q}\left(k_{0}+1\right) \geq q_{\min }$. Since

$$
(1-w) \bar{q}\left(k_{0}\right)+w\left(\frac{N K}{\sqrt{\frac{\bar{q}\left(k_{0}\right)-q_{\min }}{q_{\max }-q_{\min }} p_{\max }}}-\frac{C d}{M}\right) \leq q_{\min }+\frac{q_{\max }-q_{\min }}{p_{\max }}\left(\frac{M N K}{C d}\right)^{2},
$$

we have $\bar{q}\left(k_{0}+1\right) \leq q_{\min }+\frac{q_{\max }-q_{\min }}{p_{\max }}\left(\frac{M N K}{C d}\right)^{2}$. As 
International Journal of Bifurcation and Chaos

$$
(1-w) \bar{q}\left(k_{0}\right)+w\left(\frac{N K}{\sqrt{\frac{\bar{q}\left(k_{0}\right)-q_{\min }}{q_{\max }-q_{\min }}} p_{\max }}-\frac{C d}{M}\right)>q_{\min }+\frac{q_{\max }-q_{\min }}{p_{\max }}\left(\frac{N K}{q_{\max }+\frac{C d}{M}}\right)^{2}
$$

and

$$
(1-w) \bar{q}\left(k_{0}\right)+w\left(\frac{N K}{\sqrt{\frac{\bar{q}\left(k_{0}\right)-q_{\min }}{q_{\max }-q_{\min }} p_{\max }}}-\frac{C d}{M}\right) \leq q_{\min }+\frac{q_{\max }-q_{\min }}{p_{\max }}\left(\frac{N K}{q_{\min }+\frac{C d}{M}}\right)^{2},
$$

we have $q_{\min }+\frac{q_{\max }-q_{\min }}{p_{\max }}\left(\frac{N K}{q_{\max }+\frac{C d}{M}}\right)^{2}<\bar{q}\left(k_{0}+1\right) \leq q_{\min }+\frac{q_{\max }-q_{\min }}{p_{\max }}\left(\frac{N K}{q_{\min }+\frac{C d}{M}}\right)^{2}$. This implies that $\left(\frac{N K}{q_{\max }+\frac{C d}{M}}\right)^{2}<\frac{\bar{q}\left(k_{0}+1\right)-q_{\min }}{q_{\max }-q_{\min }} p_{\max } \leq\left(\frac{N K}{q_{\min }+\frac{C d}{M}}\right)^{2}$. This further implies that $\left(\frac{N K}{q_{\max }+\frac{C d}{M}}\right)^{2}<p\left(k_{0}+1\right) \leq\left(\frac{N K}{q_{\min }+\frac{C d}{M}}\right)^{2}$. Consequently, we have $q_{\min } \leq \frac{N K}{\sqrt{p\left(k_{0}+1\right)}}-\frac{C d}{M}<q_{\max }$. This further implies that the convex combinational of $q_{\min }$ and $\frac{N K}{\sqrt{p\left(k_{0}+1\right)}}-\frac{C d}{M}$ is larger than or equal to $q_{\min }$, that is $q_{\min } \leq(1-w) q_{\min }+w\left(\frac{N K}{\sqrt{p\left(k_{0}+1\right)}}-\frac{C d}{M}\right)$. Similarly, the convex combinational of $q_{\max }$ and $\frac{N K}{\sqrt{p\left(k_{0}+1\right)}}-\frac{C d}{M}$ is smaller than $q_{\max }$, that is $(1-w) q_{\max }+w\left(\frac{N K}{\sqrt{p\left(k_{0}+1\right)}}-\frac{C d}{M}\right)<q_{\max }$. Since $\quad q_{\max }>\bar{q}\left(k_{0}+1\right) \geq q_{\min }, \quad$ we have $\quad q_{\min } \leq(1-w) q\left(k_{0}+1\right)+w\left(\frac{N K}{\sqrt{p\left(k_{0}+1\right)}}-\frac{C d}{M}\right) \quad$ and $(1-w) q\left(k_{0}+1\right)+w\left(\frac{N K}{\sqrt{p\left(k_{0}+1\right)}}-\frac{C d}{M}\right)<q_{\max }$. In other words, we have 
International Journal of Bifurcation and Chaos

$$
\begin{gathered}
q_{\min } \leq(1-w) q\left(k_{0}+1\right)+w\left(\frac{N K}{\sqrt{p\left(k_{0}+1\right)}}-\frac{C d}{M}\right)<q_{\max }, \\
q_{\min } \leq(1-w)\left(\frac{\left(q_{\max }-q_{\min }\right) p\left(k_{0}+1\right)}{p_{\max }}+q_{\min }\right)+w\left(\frac{N K}{\sqrt{p\left(k_{0}+1\right)}}-\frac{C d}{M}\right)<q_{\max }, \\
(1-w) \frac{\left(q_{\max }-q_{\min }\right) p\left(k_{0}+1\right)}{p_{\max }}+w \frac{N K}{\sqrt{p\left(k_{0}+1\right)}}+(1-w) q_{\min }-\frac{w C d}{M}-q_{\min } \geq 0
\end{gathered}
$$

and $(1-w) \frac{\left(q_{\max }-q_{\min }\right) p\left(k_{0}+1\right)}{p_{\max }}+w \frac{N K}{\sqrt{p\left(k_{0}+1\right)}}+(1-w) q_{\min }-\frac{w C d}{M}-q_{\max }<0$. Hence, we have

$$
(1-w) \frac{\left(q_{\max }-q_{\min }\right)\left(p\left(k_{0}+1\right)\right)^{\frac{3}{2}}}{p_{\max }}+w N K+\left((1-w) q_{\min }-\frac{w C d}{M}-q_{\min }\right)\left(p\left(k_{0}+1\right)\right)^{\frac{1}{2}} \geq 0
$$

and $(1-w) \frac{\left(q_{\max }-q_{\min }\right)\left(p\left(k_{0}+1\right)\right)^{\frac{3}{2}}}{p_{\max }}+w N K+\left((1-w) q_{\min }-\frac{w C d}{M}-q_{\max }\right)\left(p\left(k_{0}+1\right)\right)^{\frac{1}{2}}<0$. This further implies that $p\left(k_{0}+1\right) \in P_{6}$ and $\bar{q}\left(k_{0}+1\right) \in Q_{6}$. Similarly, we have $\bar{q}(k) \in S_{6} \quad \forall k \geq k_{0}$. This completes the proof.

Although Lemma 4 characterizes the conditions on $\bar{q}(k) \in S_{6} \quad \forall k \geq k_{0}$ if $\bar{q}\left(k_{0}\right) \in S_{6}$, it does not guarantee the existence of a fixed point. To guarantee the existence of a fixed point, the following lemma is required:

\section{Lemma 5}

Define $\left(p^{*}, \bar{q}^{*}\right) \quad$ such that $\quad \bar{q}^{*}=\frac{N K}{\sqrt{p^{*}}}-\frac{C d}{M}=\frac{\left(q_{\max }-q_{\min }\right) p^{*}}{p_{\max }}+q_{\min } \quad . \quad$ Then $(1-w) \bar{q}^{*}+w\left(\frac{N K}{\sqrt{p^{*}}}-\frac{C d}{M}\right)=\bar{q}^{*}$

Proof:

As $(1-w) \bar{q}^{*}+w\left(\frac{N K}{\sqrt{p^{*}}}-\frac{C d}{M}\right)=(1-w)\left(\frac{N K}{\sqrt{p^{*}}}-\frac{C d}{M}\right)+w\left(\frac{N K}{\sqrt{p^{*}}}-\frac{C d}{M}\right)=\frac{N K}{\sqrt{p^{*}}}-\frac{C d}{M}=\bar{q}^{*}, \quad$ this completes the proof.

Obviously, $\left(p^{*}, \bar{q}^{*}\right)$ is a fixed point based on the dynamics defined in the sixth subset. 
International Journal of Bifurcation and Chaos

However, even though there exists a fixed point, it does not guarantee that this fixed point would be located at $S_{6}$ and satisfy Lemma 4 . Further conditions are required to be imposed and the details are discussed in following lemma. Denote the set $\Omega \equiv\left\{\bar{q}\left(k_{0}\right) \in S_{6}\right.$ : Lemma 4 is satisfied. $\}$.

\section{Lemma 6}

If

i) $\max \left(q_{\min }, q_{\min }+\frac{q_{\max }-q_{\min }}{p_{\max }}\left(\frac{N K}{q_{\min }+\frac{C d}{M}}\right)^{2}\right) \leq \bar{q}^{*}$

and

ii) $\bar{q}^{*}<\min \left(q_{\min }+\frac{q_{\max }-q_{\min }}{p_{\max }}\left(\frac{N K}{q_{\max }+\frac{C d}{M}}\right)^{2}, q_{\min }+\frac{q_{\max }-q_{\min }}{p_{\max }}\left(\frac{M N K}{C d}\right)^{2}, q_{\max }\right)$,

then

$\bar{q}^{*} \in \Omega$.

Proof:

$$
\begin{aligned}
& \text { Since } \max \left(q_{\min }, q_{\min }+\frac{q_{\max }-q_{\min }}{p_{\max }}\left(\frac{N K}{q_{\min }+\frac{C d}{M}}\right)^{2}\right) \leq \bar{q}^{*} \text { and } \\
& \bar{q}^{*}<\min \left(q_{\min }+\frac{q_{\max }-q_{\min }}{p_{\max }}\left(\frac{N K}{q_{\max }+\frac{C d}{M}}\right)^{2}, q_{\min }+\frac{q_{\max }-q_{\min }}{p_{\max }}\left(\frac{M N K}{C d}\right)^{2}, q_{\max }\right),
\end{aligned}
$$

this implies that $q_{\min } \leq \bar{q}^{*}<q_{\max }, \quad \bar{q}^{*}<\frac{q_{\max }-q_{\min }}{p_{\max }}\left(\frac{M N K}{C d}\right)^{2}+q_{\min }$ and

$$
q_{\min }+\frac{q_{\max }-q_{\min }}{p_{\max }}\left(\frac{N K}{q_{\min }+\frac{C d}{M}}\right)^{2} \leq \bar{q}^{*}<q_{\min }+\frac{q_{\max }-q_{\min }}{p_{\max }}\left(\frac{N K}{q_{\max }+\frac{C d}{M}}\right)^{2} .
$$

This further implies that $\bar{q}^{*}$ is the fixed point in $\Omega$. This completes the proof. 
International Journal of Bifurcation and Chaos

Although $\bar{q}^{*} \in \Omega$, in general it does not guarantee that $\forall \bar{q}\left(k_{0}\right) \in \Omega \quad \bar{q}\left(k_{0}\right)$ would converge to $\bar{q}^{*}$. Further conditions are required to be imposed and the details are discussed in following lemma. Define a map $\Delta V: \Omega \rightarrow \Re$ such that

$$
\begin{aligned}
& \Delta V\left(\bar{q}\left(k_{0}\right)\right) \equiv\left(\left(\frac{p_{\max }}{q_{\max }-q_{\min }}\right)^{2}+1\right)\left((1-w) \bar{q}\left(k_{0}\right)+w\left(\frac{N K}{\sqrt{\frac{\bar{q}\left(k_{0}\right)-q_{\min }}{q_{\max }-q_{\min }}} p_{\max }}-\frac{C d}{M}\right)-\bar{q}^{*}\right)^{2} \\
& -\left(\frac{\bar{q}\left(k_{0}\right)-q_{\min }}{q_{\max }-q_{\min }} p_{\max }-\frac{\bar{q}^{*}-q_{\min }}{q_{\max }-q_{\min }} p_{\max }\right)^{2}-\left(\bar{q}\left(k_{0}\right)-\bar{q}^{*}\right)^{2}
\end{aligned}
$$

and define $\max _{\bar{q}\left(k_{0}\right) \in \Omega} \Delta V\left(\bar{q}\left(k_{0}\right)\right) \equiv \Delta V^{*}$.

\section{Lemma 7}

Suppose that $\bar{q}^{*}$ satisfies the conditions in Lemma $6, \bar{q}\left(k_{0}\right) \in \Omega$ and $\Delta V^{*}<0$, then $(p(k), \bar{q}(k)) \rightarrow\left(p^{*}, \bar{q}^{*}\right)$.

Proof:

Denote $V(k) \equiv\left(p(k)-p^{*}\right)^{2}+\left(\bar{q}(k)-\bar{q}^{*}\right)^{2} \quad \forall k \geq k_{0}$. Obviously, $V(k) \geq 0 \quad \forall k \geq k_{0}$. Since $\bar{q}^{*}$ satisfies the conditions in Lemma $6, \bar{q}^{*} \in \Omega$. As 
International Journal of Bifurcation and Chaos

$$
\begin{aligned}
& V\left(k_{0}+1\right)-V\left(k_{0}\right)=\left(p\left(k_{0}+1\right)-p^{*}\right)^{2}+\left(\bar{q}\left(k_{0}+1\right)-\bar{q}^{*}\right)^{2}-\left(p\left(k_{0}\right)-p^{*}\right)^{2}-\left(\bar{q}\left(k_{0}\right)-\bar{q}^{*}\right)^{2} \\
& =\left(\frac{(1-w) \bar{q}\left(k_{0}\right)+w\left(\frac{N K}{\sqrt{p\left(k_{0}\right)}}-\frac{C d}{M}\right)-q_{\min }}{q_{\max }-q_{\min }} p_{\max }-p^{*}\right)^{2} \\
& +\left((1-w) \bar{q}\left(k_{0}\right)+w\left(\frac{N K}{\sqrt{p\left(k_{0}\right)}}-\frac{C d}{M}\right)-\bar{q}^{*}\right)^{2}-\left(p\left(k_{0}\right)-p^{*}\right)^{2}-\left(\bar{q}\left(k_{0}\right)-\bar{q}^{*}\right)^{2} \\
& =\left(\frac{(1-w) \bar{q}\left(k_{0}\right)+w\left(\frac{N K}{\sqrt{p\left(k_{0}\right)}}-\frac{C d}{M}\right)-q_{\min }}{q_{\max }-q_{\min }} p_{\max }-\frac{\bar{q}^{*}-q_{\min }}{q_{\max }-q_{\min }} p_{\max }\right)^{2} \\
& +\left((1-w) \bar{q}\left(k_{0}\right)+w\left(\frac{N K}{\sqrt{p\left(k_{0}\right)}}-\frac{C d}{M}\right)-\bar{q}^{*}\right)^{2}-\left(p\left(k_{0}\right)-\frac{\bar{q}^{*}-q_{\min }}{q_{\max }-q_{\min }} p_{\max }\right)^{2}-\left(\bar{q}\left(k_{0}\right)-\bar{q}^{*}\right)^{2} \\
& =\left(\left(\frac{p_{\max }}{q_{\max }-q_{\min }}\right)^{2}+1\right)\left((1-w) \bar{q}\left(k_{0}\right)+w\left(\frac{N K}{\sqrt{p\left(k_{0}\right)}}-\frac{C d}{M}\right)-\bar{q}^{*}\right)^{2} \\
& -\left(p\left(k_{0}\right)-\frac{\bar{q}^{*}-q_{\min }}{q_{\max }-q_{\min }} p_{\max }\right)^{2}-\left(\bar{q}\left(k_{0}\right)-\bar{q}^{*}\right)^{2} \\
& =\Delta V\left(\bar{q}\left(k_{0}\right)\right)
\end{aligned}
$$

and $\Delta V^{*}<0$, this implies that $V\left(k_{0}+1\right)-V\left(k_{0}\right)<0 \quad \forall \bar{q}\left(k_{0}\right) \in \Omega$. Since $\bar{q}\left(k_{0}\right) \in \Omega$, by the principle of the Lyapunov stability theorem, we have $(p(k), \bar{q}(k)) \rightarrow\left(p^{*}, \bar{q}^{*}\right)$. This completes the proof.

Lemma 4 to Lemma 7 characterize the conditions on both the system parameters and the initial conditions that the average queue size of the RED algorithm would converge to a fixed point in the sixth subset. As there is no fixed point in other subsets, the state vectors in these other subsets will not stay in these subsets.

\section{CONCLUSION}

This paper proposes a symbolic dynamical model of the average queue size of the RED algorithm and characterizes the conditions on both the system parameters and the initial conditions that the average queue size of the RED algorithm would converge to a fixed point. By employing 
International Journal of Bifurcation and Chaos

the symbolic dynamical system approach, both the system parameters and the initial conditions can be designed so that the average queue size of the RED algorithm would converge to a fixed point behavior.

\section{ACKNOWLWDGEMENTS}

The work obtained in this paper was supported by an Australian research grant and a research grant from the Queen Mary, University of London.

\section{REFERENCES}

[1] Priya Ranjan, Eyad H. Abed and Richard J. La, “Nonlinear instabilities in TCP-RED,” IEEE Transactions on Networking, vol. 12, no. 6, pp. 1079-1092, 2004.

[2] Priya Ranjan, Eyad H. and Abed Richard J. La, “Nonlinear instabilities in TCP-RED," The Twenty-First Annual Joint Conference of the IEEE Computer and Communications Societies, IEEE INFOCOM, vol. 1, pp. 249-258, June 23-27, 2002.

[3] L. Chen, X. F. Wang and Z. Z. Han, “Controlling bifurcation and chaos in internet congestion control model," International Symposium on Circuits and Systems, ISCAS, vol. 3, pp. III132-III135, May 25-28, 2003.

[4] J. H. C. Nga, H. H. C. Iu, B. W. K. Ling and H. K. Lam, “Analysis and control of bifurcation and chaos in average queue length in TCP/RED model,” International Journal of Bifurcation and Chaos, vol. 18, no. 8, pp. 2449-2459, 2008.

[5] Feng Liu, Zhi-Hong Guan and Hua O. Wang, "Impulsive control bifurcation and chaos in internet TCP-RED congestion control system,” The IEEE International Conference on Control and Automation, ICCA, pp. 224-227, May 30-June 1, 2007.

[6] Kirk Chang, Gitae Kim, Larry Wong, Sunil Samtani, Aristides Staikos, Mitesh Patel and Jeffrey Bowcock, "Network layer congestion control to ensure quality of service (QOS) in secure battlefield mobile ad hoc networks," The IEEE Military Communications Conference, 
International Journal of Bifurcation and Chaos MILCOM, pp. 1-7, October 29-31, 2007. 